\title{
Review of: "Role of prostate health index to predict Gleason score upgrading and high-risk prostate cancer in radical prostatectomy specimens"
}

\author{
Chi-Fai $\mathrm{Ng}^{1}$ \\ 1 The Chinese University of Hong Kong
}

Potential competing interests: The author(s) declared that no potential competing interests exist.

This was a retrospective review of a group of patients with PHI done (with some have MRI done) and followed by biopsy and then for positive case, some will have prostatectomy done the aim of study was not so clear, as the authors seem try to assess multiple primary outcomes within just 300 cases - diagnostic performance of PHI, prediction of CS upgrading etc.

I will suggest:

1. better to have one primary study aim rather than multiple and put the other in secondary outcomes

2. for pathological upgrading - it is confusing, it will depend on the initial biopsy accuracy, so for PHI it correlated with final pathology and so if the initial biopsy (by luck or so) got the right spot and have the accurate result, then it will be "no upgrading" - so it is not just depend on PHI to determine upgrading or not. Clinically, more important is for patient in low risk, such as G3+3, G3+4, who may go for AS or focal therapy. Therefore, maybe they could focus on $\mathrm{G} 3+3 / 3+4$ at biopsy who has or has not upgraded during prostatectomy 